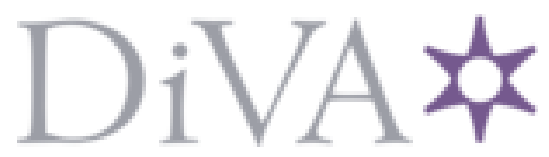

http://www.diva-portal.org

This is the published version of a paper published in Journal of Geophysical Research.

Citation for the original published paper (version of record):

Hamrin, M., Marghitu, O., Norqvist, P., Buchert, S., André, M. et al. (2012)

The role of the inner tail to midtail plasma sheet in channeling solar wind power to the ionosphere.

Journal of Geophysical Research, 117: A06310

http://dx.doi.org/10.1029/2012JA017707

Access to the published version may require subscription.

N.B. When citing this work, cite the original published paper.

Permanent link to this version:

http://urn.kb.se/resolve?urn=urn:nbn:se:umu:diva-56973 


\title{
The role of the inner tail to midtail plasma sheet in channeling solar wind power to the ionosphere
}

\author{
M. Hamrin, ${ }^{1}$ O. Marghitu, ${ }^{2}$ P. Norqvist, ${ }^{1}$ S. Buchert, ${ }^{3}$ M. André, ${ }^{3}$ B. Klecker, ${ }^{4}$ \\ L. M. Kistler, ${ }^{5}$ and I. Dandouras ${ }^{6}$ \\ Received 12 March 2012; revised 25 April 2012; accepted 25 April 2012; published 7 June 2012.
}

[1] In this article we use Cluster power density $(\mathbf{E} \cdot \mathbf{J})$ data from 2001, 2002, and 2004 to investigate energy conversion and transfer in the plasma sheet. We show that a southward IMF $B_{z}$ is favorable for plasma sheet energy conversion, and that there is an increased particle and Poynting flux toward the Earth at times when Cluster observes an enhanced energy conversion in the plasma sheet. Conversion from electromagnetic to kinetic energy is increasingly dominant farther down-tail, while the generation of electromagnetic power from kinetic energy becomes important toward the Earth with a maximum at roughly $10 R_{E}$. By linking observations of the key quantity $\mathbf{E} \cdot \mathbf{J}$ to observations of the solar wind input and earthward energy flux, our results demonstrate the role of the inner tail to midtail plasma sheet as a mediator between the solar wind energy input into the magnetosphere and the auroral dissipation in the ionosphere.

Citation: Hamrin, M., O. Marghitu, P. Norqvist, S. Buchert, M. André, B. Klecker, L. M. Kistler, and I. Dandouras (2012), The role of the inner tail to midtail plasma sheet in channeling solar wind power to the ionosphere, J. Geophys. Res., 117, A06310, doi:10.1029/2012JA017707.

\section{Introduction}

[2] In the literature, there are many investigations suggesting that the plasma sheet energy conversion acts as an important mediator between the solar wind (SW) energy input into the magnetosphere and the auroral dissipation in the ionosphere. For example, high speed plasma flows in the plasma sheet have been shown to correlate well both with the orientation of the interplanetary magnetic field (IMF) [Juusola et al., 2011] and with auroral activity as measured by the AE index [Angelopoulos et al., 1994]. The results of these studies implicitly suggest a general energy transfer scheme from the SW, to the plasma sheet, and then to the ionosphere. However, to our knowledge, there is a lack of investigations directly linking the SW energy input and the energy flux toward the ionosphere to observations of the plasma sheet power density. The power density, $\mathbf{E} \cdot \mathbf{J}$ (where $\mathbf{E}$ is the electric field and $\mathbf{J}$ the current density), is a key quantity when investigating energy conversion issues. $\mathbf{E} \cdot \mathbf{J}$ corresponds to the amount of energy (per unit volume and per unit time) converted between its electromagnetic and

\footnotetext{
${ }^{1}$ Department of Physics, Umeå University, Umeå, Sweden.

${ }^{2}$ Institute for Space Sciences, Bucharest, Romania.

${ }^{3}$ Swedish Institute of Space Physics, Uppsala, Sweden.

${ }^{4}$ Max-Planck-Institut für Extraterrestrische Physik, Garching, Germany.

${ }^{5}$ Space Science Center, University of New Hampshire, Durham, New Hampshire, USA.

${ }^{6}$ IRAP, UPS-OMP, University of Toulouse, CNRS, Toulouse, France.

Corresponding author: M. Hamrin, Department of Physics, Umeå University, SE-901 87 Umeå, Sweden. (hamrin@space.umu.se)

C2012. American Geophysical Union. All Rights Reserved.
}

kinetic forms. A value $\mathbf{E} \cdot \mathbf{J}<0$ indicates a generator, in which kinetic energy is converted to electromagnetic energy, and the electromagnetic energy density hence increases. For a load, $\mathbf{E} \cdot \mathbf{J}>0$, and energy is transferred in the opposite direction. The interpretation of $\mathbf{E} \cdot \mathbf{J}$ can be verified from the Poynting theorem

$$
\frac{\partial W_{E M}}{\partial t}=-\nabla \cdot \mathbf{S}-\mathbf{E} \cdot \mathbf{J}
$$

where $W_{E M}$ is the electromagnetic energy density, and $\mathbf{S}$ is the Poynting vector.

[3] Due to the cross-tail current, and the dawn-dusk electric field, the plasma sheet corresponds to a load at large scales, converting electromagnetic energy to kinetic (thermal and plasma bulk) energy. On smaller scales, however, the plasma sheet shows a high level of fine structure in the energy conversion, hosting both load and generator regions. This complicated structure of the plasma sheet energy conversion has been verified both from numerical simulations [e.g., Birn and Hesse, 2005; Tanaka et al., 2010] and from spacecraft observations [e.g., Hamrin et al., 2009]. In Figure 1 we present a simple cartoon of the major energy transfer processes of the Earth's magnetosphere. It shows the plasma sheet as an important link in the long chain of processes eventually causing, e.g., auroral activity at lower altitudes.

[4] To our knowledge, the first experimental investigations of generator regions in the plasma sheet were presented in Marghitu et al. [2006], Hamrin et al. [2006], and Rosenqvist et al. [2006]. However, it is only recently that the overall behavior of the plasma sheet energy conversion has been investigated more intensely. Using Cluster $\mathbf{E} \cdot \mathbf{J}$ data 


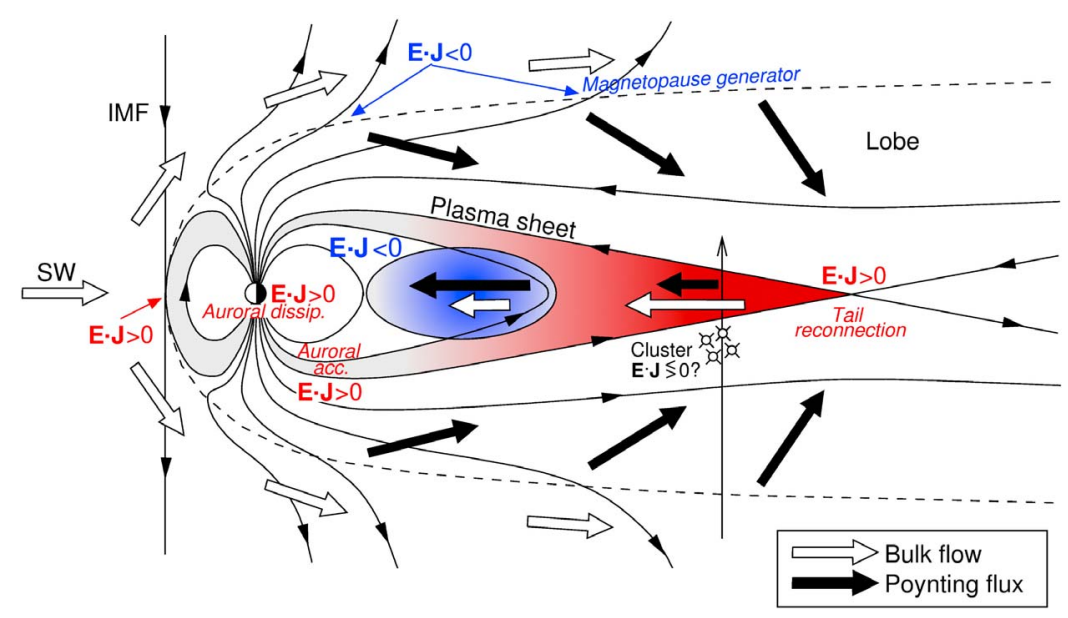

Figure 1. A schematic cartoon showing some main features of the magnetospheric energy budget. See the text for details.

from several years, Hamrin et al. [2011] found that loads and generators regularly exist in the plasma sheet probed by Cluster. Load regions appear to be approximately three times as common as generator regions, and loads are generally observed closer to the neutral sheet, while generators often prefer locations toward the plasma sheet boundary layer (PSBL). In previous investigations, variations in $\mathbf{E} \cdot \mathbf{J}$ have also been linked to, e.g., earthward flowing plasmoids and magnetic flux ropes [Lui et al., 2007; Zong et al., 2007].

[5] In this article we use Cluster data for discussing the role of the inner tail to midtail plasma sheet for the energy conversion between electromagnetic and kinetic energy forms, and for the energy transfer toward the ionosphere. To probe the energy conversion we will use the key quantity of the power density, $\mathbf{E} \cdot \mathbf{J}$. The multispacecraft Cluster mission [Escoubet et al., 2001] is particularly favorable for observational investigations of the energy conversion. The reason is that at least four simultaneous magnetic field measurements (provided by the four Cluster spacecraft) are needed for estimating the current density $\mathbf{J}$.

[6] In section 2 we discuss relevant energy conversion and transfer processes in the magnetosphere. Section 3 contains a short overview of the data and methods used. In section 4.1 we analyze the response of the plasma sheet energy conversion in relation to the SW energy input (as probed by the IMF $B_{z}$ direction). The relation between the plasma sheet energy conversion and the energy transfer toward lower altitudes (via BBFs and Poynting flux) is investigated in section 4.2. Our results are summarized in section 5.

\section{Energy Conversion and Transfer in the Inner Tail to Midtail Plasma Sheet}

[7] The SW kinetic energy flux is the major energy source for the magnetospheric energy budget. This flux is about 20 times larger than the SW Poynting flux at the distance of the Earth's orbit, but only a fraction of the available SW kinetic energy is needed to power the magnetospheric dynamics [Koskinen and Tanskanen, 2002]. Magnetic reconnection [Paschmann et al., 1979] is a key process for controlling energy transfer into and within the magnetosphere [Dungey, 1961]. Locally, reconnection corresponds to a load where free magnetic energy is transferred into accelerated particles and heat.

[8] Reconnection controls the transfer of SW energy across the magnetopause [Pulkkinen et al., 2007]. The energy input depends foremost on the IMF direction and the SW speed [Pulkkinen et al., 2008; Palmroth et al., 2010]. For southward IMF, reconnection is expected to occur at the dayside magnetopause. The reconnected field lines are dragged down-tail by the SW, and in regions beyond the cusp, the magnetic tangential force acts against the SW flow, which causes energy from the SW to be transferred into magnetic energy density of the lobe magnetic field [Siscoe and Cummings, 1969]. Such magnetopause generators have recently been investigated by, e.g., Rosenqvist et al. [2008] and Palmroth et al. [2010].

[9] The energy stored in the tail magnetic field is released through tail reconnection [see, e.g., Sharma et al., 2008, and references therein]. A near-Earth neutral line (NENL) is expected to form $20-30 R_{E}$ down-tail in the plasma sheet during substorm expansion [Nishida et al., 1981]. The energy is transported away from the reconnection site as high speed plasma flows toward the ionosphere in the coupled magnetosphere-ionosphere (M-I) system, as well as into interplanetary space. For a long time, flows in the plasma sheet were considered to be rather smooth and laminar, but now we know that the plasma sheet is much more structured and complicated than initially anticipated. In the early $90 \mathrm{~s}$, bursty bulk flows (BBFs) were discovered, and soon it was observed that BBFs are commonly occurring in the plasma sheet [Angelopoulos et al., 1992]. Typically, BBFs are expected to be caused by reconnection, and they correspond to bursty high speed flow events observed on a 10 min timescale, and composed of individual flow burst $(\gtrsim 400 \mathrm{~km} / \mathrm{s})$ on shorter timescales [e.g., Scholer et al., 1984; Angelopoulos et al., 1992; Chen and Wolf, 1993]. While observed about a tenth of the time in the plasma sheet, they contribute approximately to half of the circulated mass, energy, and flux [Sergeev, 2005]. BBFs are related to auroral phenomena at the ionospheric end of the M-I system, e.g., auroral expansions, localized brightenings, and auroral streamers [e.g., Amm and Kauristie, 2002; Sergeev, 2005]. 
[10] The energy transferred into the magnetosphere is eventually dissipated through various processes such as ring current dissipation, auroral Joule heating, and charged particle precipitation into the ionosphere. It has been found that the plasma sheet dissipates (in the form of plasma heating and plasmoid ejection) comparable amounts of energy during substorms [Ieda et al., 1998; Slavin et al., 1993]. The energy conversion in the plasma sheet is hence an important factor for the magnetospheric energy budget.

[11] Processes important for both the energy and mass transport within the coupled M-I system, as well as for the ultimate energy dissipation in the auroral ionosphere, have been investigated extensively. See for example Paschmann et al. [2002] for a review. Recent investigations suggest that energy conversion regions (ECRs) in the plasma sheet, in the form of both loads and generators, are often associated with BBFs [Hamrin et al., 2011; Marghitu et al., 2010; O. Marghitu et al., manuscripts in preparation, 2012]. The results from Hamrin et al. [2011] suggest a rather complicated structure of the plasma sheet energy conversion, with the possibility of multiple conversion back and forth between the kinetic (thermal and plasma bulk) and electromagnetic energy forms before the energy eventually is dissipated in the ionosphere.

[12] Electromagnetic power generation and dissipation along auroral field lines was addressed by Angelopoulos et al. [2002]. By using conjugated data from the Polar and Geotail satellites, they observed that the earthward particle energy flux is about ten times as large as the electromagnetic energy flux at the same altitude. Moreover, most electromagnetic energy density generated at $\sim 18 R_{E}$ is found to be dissipated before it reaches the Polar altitude of $\sim 5 R_{E}$ (above the auroral acceleration region). However, the Poynting flux observed by Polar is still more than enough for explaining the auroral particle acceleration below the spacecraft. Moreover, even if only a small part of the BBF energy couples to Alfvén waves and is radiated away, it is nonetheless sufficient for powering the auroral acceleration during substorms. The results presented by Angelopoulos et al. [2002] indicate that there must exist mechanisms corresponding to the loss of electromagnetic energy along the field lines, and BBF-generated Alfvén waves were suggested as a possible candidate. Comparing with the results of Hamrin et al. [2011], we note that such an electromagnetic energy conversion mechanism could well be described by the existence of ECRs along the field lines between the reconnection site (load) and the auroral acceleration and ionospheric dissipation (loads).

\section{Method and Data}

[13] In this investigation we use Cluster data from 2001, 2002, and 2004 to investigate the plasma sheet energy conversion and transfer trough the evaluation of $\mathbf{E} \cdot \mathbf{J}$. For these years, the Cluster spacecraft configuration was favorable (approximately an equilateral tetrahedral with scale size $1000-4000 \mathrm{~km}$ ). During 2003, the scale size was of the order of, or smaller, than the typical ion gyro radius, while the configuration was in a multiscale mode in 2005 and later years. Magnetic field measurements from the FGM magnetometer instrument are used for obtaining the current density by using the curlometer method. The electric field is derived from the two CIS ion instruments, CODIF and HIA, on the assumption that the $\mathbf{E} \times \mathbf{B}$ drift is dominant. Electric field estimates from the EFW double-probe instrument are used for double-checking the results. For a detailed discussion of the Cluster mission and instruments, see Escoubet et al. [2001, and references therein].

[14] Near the Earth (in the inner tail to midtail plasma sheet) the space plasma is mostly well described by ideal MHD, where $\mathbf{E}+\mathbf{v} \times \mathbf{B}=0$, and the $\mathbf{E}$ parallel to the magnetic field, $E_{\|}$, is assumed to be zero. Then $\mathbf{E} \cdot \mathbf{j}=\mathbf{E}_{\perp} \cdot \mathbf{j}_{\perp}$. This assumption can break down in small regions, where then also the term $E_{\|} \cdot j_{\|}$potentially contributes to $\mathbf{E} \cdot \mathbf{j}$. In this study we use the ion velocity $\mathbf{v}$ and $\mathbf{B}$ measured by instruments on satellites to estimate $\mathbf{E}_{\perp}$, and cannot directly detect any regions where $E_{\|} \neq 0$. However, considering the timescale of our ion measurements, of about $10 \mathrm{~s}(\approx 4 \mathrm{~s})$, this contribution is likely to be negligible most of the time. We therefore assume that the contributions of $E_{\|} \cdot j_{\|}$to the overall power density is not significant, and we neglect it.

[15] The power density is evaluated in the GSM frame of reference. Picking a correct reference frame is very important since the power density is not invariant under coordinate transformations. The choice of frame should be based on the relevant energy conversion processes within the system, e.g., (a) the reconnection X-line load, (b) load and generator regions within the highly structured plasma sheet (possibly related to BBFs), (c) the load in the auroral acceleration region, and (d) the load corresponding to the ionospheric Joule heating. As discussed in Hamrin et al. [2011], the GSE/GSM frame is the optimal one in our case.

[16] Using the $\mathbf{E} \cdot \mathbf{J}$ data we have identified 555 ECRs from the years of interest. The identification is made by an automatic routine, and only clearly identifiable regions with significant $|\mathbf{E} \cdot \mathbf{J}|>0$ above the surrounding fluctuations are selected. To be accepted by the routine, every event must fulfil a set of instrumental and physical criteria. In total, 428 Concentrated Load Regions (CLRs) and 127 Concentrated Generator Regions (CGRs) have been identified. In addition to this, we have randomly selected almost 2500 reference events with random time extent between $100 \mathrm{~s}$ and $500 \mathrm{~s}$. The only selection criterion for these RAND events is that the spacecraft instruments are fully operational. There are no extra requirements on, e.g., measurable energy conversion. The RAND events are expected to capture the general behavior of the plasma sheet energy conversion. For a discussion about the data, the selection routine, and a more theoretical motivation for the use of the power density quantity, we refer to Hamrin et al. [2011, and references therein].

[17] In this article we analyze the occurrence of ECRs in relation to the north-south direction of IMF $B_{z}$, to the plasma sheet bulk flow, and to the Poynting flux. For the IMF investigation, we use $64 \mathrm{~s}$ averages of the ACE SW data [Chiu et al., 1998; Smith et al., 1998] time shifted to the nominal magnetopause $\left(10 R_{E}\right)$. The plasma bulk flow is obtained from the HIA and CODIF instruments (with the assumption that all ions are $\mathrm{H}+$ ). In the computation of the Poynting flux, the background magnetic field (a polynomial fit) has been subtracted from the total magnetic field to obtain the wave contribution. However, no background electric field has been subtracted. To be consistent with the 


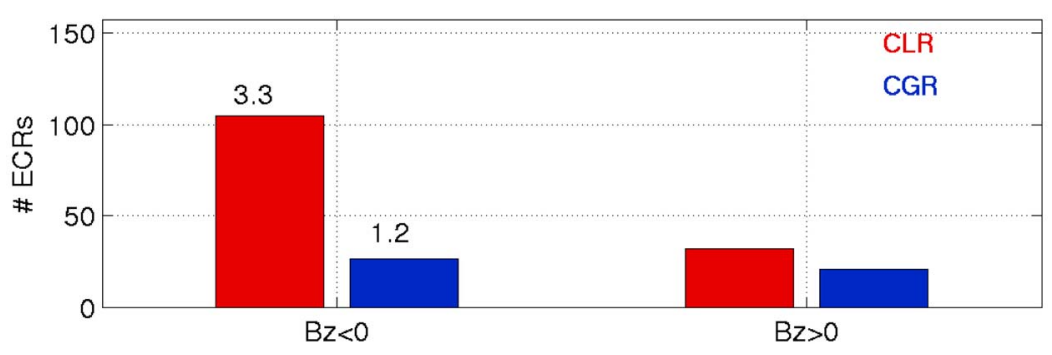

Figure 2. The occurrence of plasma sheet load (red) and generator (blue) regions for southward $\left(B_{z}<0\right)$ and northward $\left(B_{z}>0\right) \mathrm{IMF}$, respectively. We only include events which correspond to a constant southward or northward IMF during an extended 60 min interval before the starting time of the ECRs. No extra time delay between variations in the IMF and the ECR response is assumed. The ratio between the number of ECRs with $B_{z}<0$ and $B_{z}>0$ is indicated at the top of the $B_{z}<0$ bar.

power density calculation, the Poynting flux has been computed in the same frame of reference as $\mathbf{E} \cdot \mathbf{J}$, i.e., GSM.

\section{Observations}

\subsection{ECR Response to SW Input}

[18] SW energy input is known to be efficient under conditions of southward IMF $B_{z}$, even though investigations show that reconnection also operates during northward IMF, but at higher latitudes [e.g., Reiff and Burch, 1985]. In this section we investigate the response in the plasma sheet energy conversion with respect to variations in the GSM IMF $B_{z}$ component.

[19] It is reasonable to assume that the plasma sheet energy conversion is not responding instantaneously to variations in the IMF. The histogram in Figure 2 therefore shows the occurrence of ECRs for conditions of steady northward and southward IMF $B_{z}$ during intervals $\geq 60 \mathrm{~min}$ before the starting time of the ECRs. The timescale of $60 \mathrm{~min}$ is reasonable for including a possible time lag between the SW energy input and the plasma sheet response [see, e.g., Bargatze et al., 1986]. The ratio between the number of events with $B_{z}<0$ and $B_{z}>0\left(N_{B_{z}<0} / N_{B_{z}>0}\right)$ is indicated at the top of the CLR and CGR bars. We see that CLRs and CGRs are observed during southward IMF 3.3 and 1.2 times as often as during northward IMF, and we find confirmed that a southward IMF is favorable for plasma sheet energy conversion at Cluster altitudes.

[20] The response to the IMF $B_{z}$ sign is seen to be stronger for CLRs than for CGRs. This effect could be traced back to variations in the CLR and CGR spatial distributions in $x y z$ space (Figure 3 ) as probed by Cluster, and in relation to the general plasma sheet motion, thinning, and expansion during the substorm cycle. Note that loads can be expected to exist for example rather close to the reconnection site where the plasma is accelerated, while generators may well be more common closer to the inner boundary of the plasma sheet, in the region of braking and diversion of earthward plasma flows. In addition to this, we may also expect that loads and generators may appear in different regions of the BBF channels (e.g., the front side, rear side or the flanks).

[21] In Figure 3a we indicate the expected occurrence of plasma sheet loads and generators as a general function of $x$. A schematical Cluster orbit is shown in Figure 3b. Due to properties of the orbit, plasma sheet data from various regions in $x y z$ space (from regions closer to the neutral sheet as well as from regions in the PSBL and closer to the flanks) are included in this investigation - see also Hamrin et al. [2011] for a discussion of the $x y z$ distributions of CLRs and CGRs. For $x$ values below $\sim 14 R_{E}$, Cluster data are obtained near the flanks. For larger distances, we may expect that Cluster in general probes the plasma sheet near the NENL, in regions where we are more close to the maximum of the load distribution, but toward the declining tail of the generator distribution. Consequently, the data in this investigation can be used to verify the difference between the spatial distributions of CLRs and CGRs in $x y z$ space as probed by Cluster.

[22] After the discussion above we can also explain why CGRs respond more weakly to IMF $B_{z}$ than CLRs (see Figure 2). The general magnetotail configuration is expected to vary with, e.g., the SW input and the substorm cycle. After prolonged intervals of southward IMF we may expect a substorm expansion, with a resulting tail-ward motion of the NENL [Baumjohann et al., 1999]. A few minutes before onset, reconnection occurs at $x$ approximately between $-16 R_{E}$ and $-20 R_{E}$ [Miyashita et al., 2009], i.e. in the region of the Cluster plasma sheet crossings in 2001, 2002, and 2004. (Note that Cluster mostly probes the earthward side of the NENL according to our data - see the discussion in relation to Figure 4 below.) The relative position between the NENL and the Cluster spacecraft will vary during the substorm cycle. On the average, Cluster is probing regions near the NENL (generally on the earthward side), i.e. regions which are dominated by earthward plasma acceleration (at the peak of the CLR distribution and the tail of the CGR distribution in Figure 3a). The location of the NENL depends on the SW [Nagai, 2006], and it has been shown that the neutral line occurs closer to the Earth under strong solar wind conditions [Miyashita et al., 2009]. For such conditions, this would hence push the Cluster plasma sheet crossings further toward an increasing (a decreasing) CLR (CGR) distribution as shown in Figures 3a-3b, i.e., toward an even weaker response of the CGRs. In summary, the Cluster location close to the peak (tail) of the CLR (CGR) distribution, together with the magnetotail dynamics with respect to the IMF and the substorm cycle, could explain why the occurrence of CGRs seems to be less affected by the IMF $B_{z}$ direction. 
a)

b)
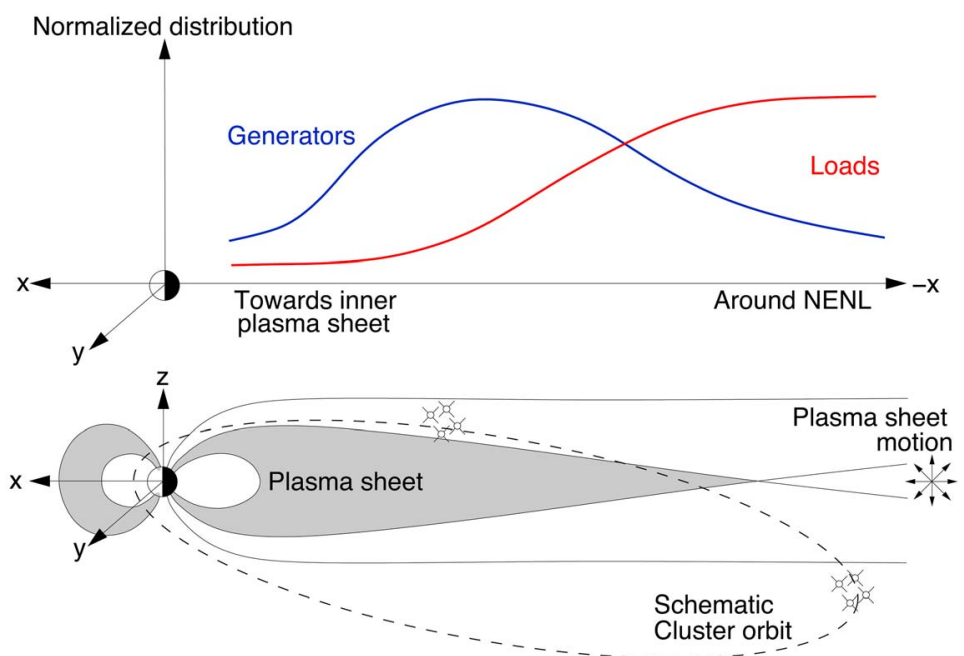

c)

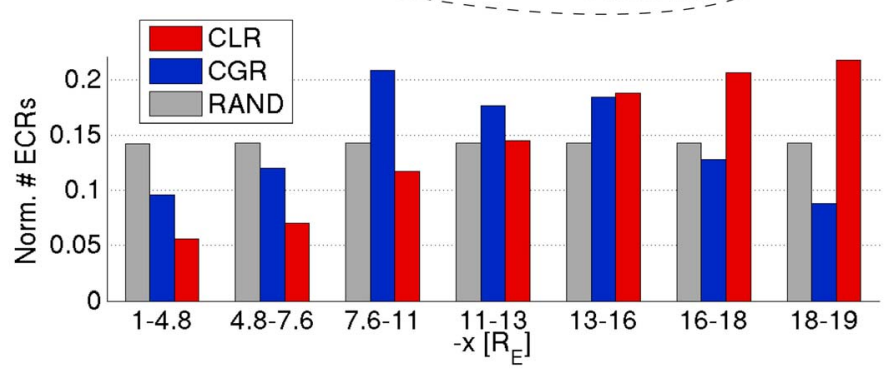

Figure 3. (a) Schematic variation of the occurrence of load and generator regions in the plasma sheet. (b) Schematic Cluster orbit. Note that effects from the Cluster orbit imply that a rather extended region in GSM $x y z$ space is probed. (c) Observed occurrence of CLRs, CGRs, and RAND events versus GSM $-x$.

[23] In Figure 3c we present the occurrence of CLRs, CGRs, and RAND events as a function of GSM $-x$. The data are normalized to one and binned so that there is an equal sampling between the bins (the same number of RAND events in all bins). We see that the expected variation in the spatial distribution of CLRs and CGRs is indeed observed, with CGRs generally occurring closer to the inner boundary of the plasma sheet. Presenting the CLR and CGR distributions only over the $\mathrm{x}$ coordinate rather than in full 2D or 3D space does perhaps not give us the complete picture, since, for example, data obtained at smaller $x$ tend to origin from regions closer to the flanks or the PSBL. However, this does not change the general conclusion that spatial $(x y z)$ variations in the CLR and CGR distributions affect the obtained IMF response as displayed in Figure 2.

\subsection{Relation Between ECRs and Energy Transfer}

[24] Figures $4 a-4 c$ show the variation of the plasma sheet energy conversion in relation to (a) the observed plasma flow in the GSM $x$ direction $\left(V_{x}\right)$, (b) the total plasma flow $\left(\left|V_{\text {tot }}\right|\right)$, and (c) the Poynting flux along the GSM $x$ direction $\left(S_{x}\right)$. Note that the horizontal axis is reversed so that positive values are to the left. The third bin from the left in all panels corresponds to small values $\left(-50 \leq V_{x}<50 \mathrm{~km} / \mathrm{s}\right.$, $\left|V_{\text {tot }}\right|<50 \mathrm{~km} / \mathrm{s}$, and $\left.-0.05 \leq S_{x}<0.05 \mu \mathrm{W} / \mathrm{m}^{2}\right)$.

[25] From Figure 4a we see that the plasma sheet flow in the $x$ direction typically is rather low - see the large gray RAND data bar in the third bin from the left. From the RAND reference data set it can also be seen that Cluster more often observes plasma flows toward the Earth $\left(V_{x}>0\right)$ than away from the Earth $\left(V_{x}<0\right)$. This is consistent with the Cluster satellites generally probing regions earthward of the NENL during the years of interest. Moreover, since the CLR and CGR bars dominate in the two leftmost bins, we note that both CLRs and CGRs are associated with high speed flows, $V_{t o t}$, especially toward the Earth.

[26] It should be noted that we mainly study in this section the correlation between the ECR occurrence and the energy transfer along the GSM $x$ direction. Detailed investigations regarding the energy transfer in full $3 \mathrm{D}$ space is outside the scope of the present article, but it is the focus for a future investigation. However, our data show that the $V_{x}$ component often gives a dominant contribution to the total plasma flow velocity when ECRs are observed, even though there are several ECRs where the $V_{y}$ and/or $V_{z}$ contribution cannot be neglected. This can be verified from Figure $4 \mathrm{~b}$ where we show the ECR occurrence versus $\left|V_{\text {tot }}\right|$.

[27] Analyzing the RAND reference data set in Figure 4c, we see that the $x$ component of the Poynting flux is dominantly directed toward the Earth. This is consistent with the expectation that Cluster probes regions earthward of the NENL, and that a large part of the energy is ultimately dissipated in the ionosphere. Moreover, typical values of the RAND data are small, $-0.05 \leq S_{x}<0.05 \mu \mathrm{W} / \mathrm{m}^{2}$. CLRs and CGRs, on the other hand, are often correlated with a stronger Poynting flux toward the Earth. The Poynting flux observed by Cluster is likely to be a superposition, originating from both local and distant sources. Preliminary event studies 
a)

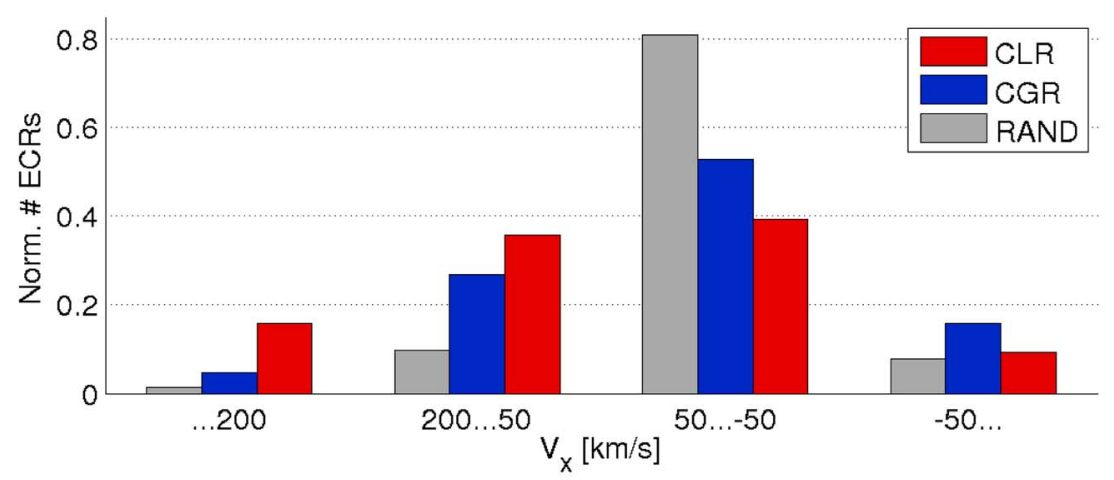

b)

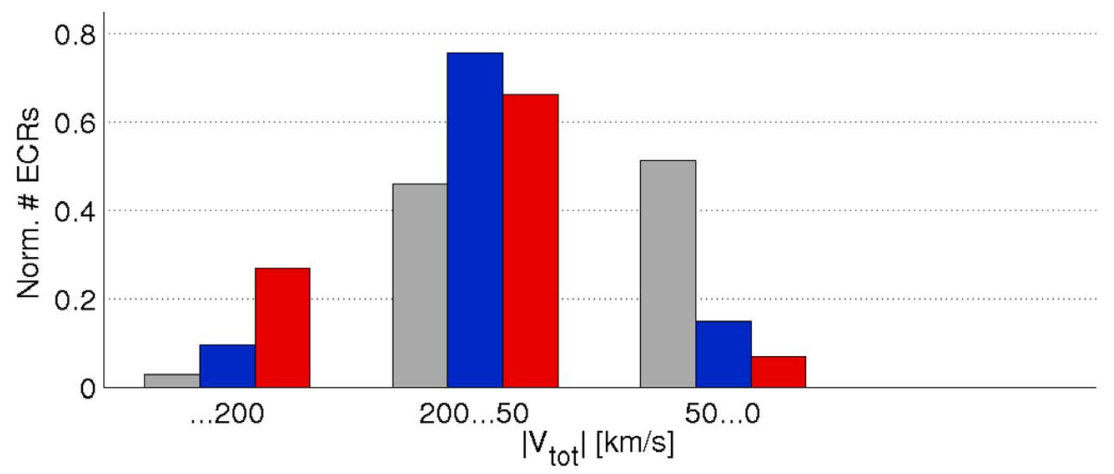

c)

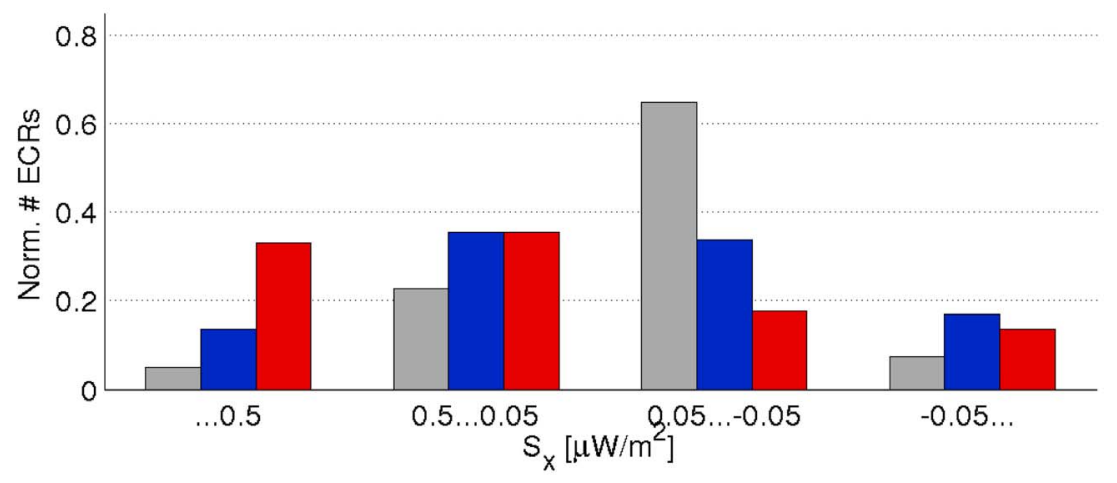

Figure 4. CLR, CGR, and RAND occurrence versus (a) plasma flows $V_{x}$, (b) $\left|V_{\text {tot }}\right|$, and (c) Poynting flux $S_{x}$. Note that the $V_{x}$ and $S_{x}$ axes are reversed so that positive values (fluxes/flows toward the Earth) are to the left.

[Constantinescu et al., 2010] suggest that conditions inside ECRs favor the generation of low frequency waves, while plasma is much more stable outside these regions. Although in the explored cases the detected waves originated from both types of ECRs, the local component appeared to be stronger for CLRs. In order to check if these features represent general properties of the ECRs, a statistical investigation is planned for a future study.

\section{Summary and Discussion}

[28] The plasma sheet energy conversion has recently been investigated in detail through the analysis of the key quantity, $\mathbf{E} \cdot \mathbf{J}$ [see, e.g., Hamrin et al., 2011, and references therein]. Through these investigations, it has been shown that spacecraft estimates of $\mathbf{E} \cdot \mathbf{J}$ can indeed be used for probing the plasma sheet energy conversion. By using
Cluster observations in the plasma sheet at a geocentric distance of about 15-20 $R_{E}$, Hamrin et al. [2011] have compiled and presented a consistent picture of the energy conversion between the electromagnetic and kinetic energy forms. Issues such as the occurrence, location, size, and strength of the energy conversion, as well as a likely relation to auroral activity at lower altitudes, have been addressed. The results are consistent with large scale MHD simulations [Birn and Hesse, 2005].

[29] ECRs are suggested to be related to BBFs [Marghitu et al., 2010; Hamrin et al., 2011]. In the present investigation we have mainly considered the relation between ECRs and the plasma flow in the GSM $x$ direction, but as mentioned in section 4.2, contributions from $V_{y}$ and $V_{z}$ may also be important. Even though we expect a relation between ECRs and BBFs, preliminary investigations have shown that far from all BBFs are related to ECRs. On the other hand, a 
majority of the ECRs are observed in correlation with significant plasma flows $V_{\text {tot }}$ (see Figure 4b). Only about $10 \%$ of the identified ECRs are observed under slow flow conditions. This is significantly different from the RAND events, which are more often correlated with slow flows. The cause for the slow flow CLRs and CGRs need to be analyzed thoroughly. Investigations of this issue as well as the detailed relationship between ECRs and BBFs are, however, outside the scope of the present article, but is planned for a future study.

[30] In the present investigation we add to the acquired knowledge about the plasma sheet energy conversion by including information about the role of the plasma sheet in transferring energy, originally coming from the SW, toward the Earth's ionosphere. We use Cluster data to show that a southward IMF $B_{z}$ is favorable for plasma sheet energy conversion. We also show that there is an energy flow (both as Poynting flux and as plasma flows) toward the Earth. Our data confirm that at the tail-ward end of the investigated region (down-tail to about $20 R_{E}$ ) power conversion from electromagnetic to kinetic and thermal power is dominant, while near the Earthward end this kinetic power is partially converted back to Poynting flux, from where it would then be available to power nightside auroral activity. The correlation between the plasma sheet $\mathbf{E} \cdot \mathbf{J}$ and auroral activity was discussed in Hamrin et al. [2011]. They found that the occurrence frequency of CLRs and CGRs increases with increasing geomagnetic activity as measured by the Kp and $\mathrm{AE}$ indices. Detailed investigations of conjugate events based on high-altitude Cluster data and low-altitude FAST data, suggest as well a strong relationship between CGR observations by Cluster and auroral activity probed by FAST [Hamrin et al., 2006].

[31] To our knowledge, this is the first time that actual responses in the plasma sheet $\mathbf{E} \cdot \mathbf{J}$ have been linked to the SW energy input at the magnetopause and the energy transfer in the plasma sheet toward the ionosphere. Since a minimum of four simultaneous measurements of the magnetic field are needed for estimating the full current density, thorough investigations of $\mathbf{E} \cdot \mathbf{J}$ have not been possible until the multispacecraft Cluster mission was available (last $\sim 10$ years).

[32] Acknowledgments. We thank the FGM, CIS, and EFW teams for providing Cluster data. O.M. acknowledges support through the PECS contract ECSTRA, C98048. We thank L. Andersson, H. Nilsson, T. Karlsson, and K. Rönnmark for fruitful discussions.

[33] Robert Lysak thanks the reviewers for their assistance in evaluating this paper.

\section{References}

Amm, O., and K. Kauristie (2002), Ionospheric signatures of bursty bulk flows, Surv. Geophys., 23(1), 1-32, doi:10.1023/A:1014871323023.

Angelopoulos, V., W. Baumjohann, C. Kennel, F. Corotiti, M. Kivelson, R. Pellat, R. Walker, H. Luhr, and G. Paschmann (1992), Bursty bulk flows in the inner central plasma sheet, J. Geophys. Res., 97(A4), 4027-4039.

Angelopoulos, V., C. F. Kennel, F. V. Coroniti, R. Pellat, M. G. Kivelson, R. J. Walker, C. T. Russell, W. Baumjohann, W. C. Feldman, and J. T. Gosling (1994), Statistical characteristics of bursty bulk flow events, J. Geophys. Res., 99(A11), 21,257-21,280.

Angelopoulos, V., J. A. Chapman, F. S. Mozer, J. D. Scudder, C. T. Russell, K. Tsuruda, T. Mukai, T. J. Hughes, and K. Yumoto (2002), Plasma sheet electromagnetic power generation and its dissipation along auroral field lines, J. Geophys. Res., 107(A8), 1181, doi:10.1029/2001JA900136.
Bargatze, L. F., D. N. Baker, and R. L. McPherron (1986), Magnetospheric response to solar wind variations, in Solar Wind Magnetosphere Coupling, Astrophys. Space Sci. Libr., vol. 126, edited by Y. Kamide and J. A. Slavin, pp. 93-100, D. Reidel, Dordrecht, Netherlands.

Baumjohann, W., M. Hesse, S. Kokubun, T. Mukai, T. Nagai, and A. Petrukovich (1999), Substorm dipolarization and recovery, J. Geophys. Res., 104(A11), 24,995-25,000, doi:10.1029/1999JA900282.

Birn, J., and M. Hesse (2005), Energy release and conversion by reconnection in the magnetotail, Ann. Geophys., 23(10), 3365-3373.

Chen, C., and R. Wolf (1993), Interpretation of high-speed flows in the plasma sheet, J. Geophys. Res., 98(A12), 21,409-21,419.

Chiu, M., et al. (1998), ACE spacecraft, Space Sci. Rev., 86(1-4), 257-284.

Constantinescu, O., O. Marghitu, M. Hamrin, and K. Glassmeier (2010), Low frequency waves in and around energy conversion regions in the plasma sheet, Eos Trans. AGU, 91(26), Meet. Am. Suppl., Abstract SM43A-06.

Dungey, J. (1961), Interplanetary magnetic field and auroral zones, Phys. Rev. Lett., 6(2), 47-48.

Escoubet, C. P., M. Fehringer, and M. Goldstein (2001), The Cluster mission-Introduction, Ann. Geophys., 19, 1197-1200.

Hamrin, M., et al. (2006), Observations of concentrated generator regions in the nightside magnetosphere by Cluster/FAST conjunctions, Ann. Geophys., 24(2), 637-649.

Hamrin, M., P. Norqvist, O. Marghitu, S. Buchert, B. Klecker, L. M. Kistler, and I. Dandouras (2009), Occurrence and location of concentrated load and generator regions observed by Cluster in the plasma sheet, Ann. Geophys., 27(11), 4131-4146.

Hamrin, M., O. Marghitu, P. Norqvist, S. Buchert, M. André, B. Klecker, L. M. Kistler, and I. Dandouras (2011), Energy conversion regions as observed by Cluster in the plasma sheet, J. Geophys. Res., 116, A00K08, doi:10.1029/2010JA016383. [Printed 117(A1), 2012.]

Ieda, A., S. Machida, T. Mukai, Y. Saito, T. Yamamoto, A. Nishida, T. Terasawa, and S. Kokubun (1998), Statistical analysis of the plasmoid evolution with Geotail observations, J. Geophys. Res., 103, 4453-4465, doi:10.1029/97JA03240.

Juusola, L., N. Østgaard, and E. Tanskanen (2011), Statistics of plasma sheet convection, J. Geophys. Res., 116, A08201, doi:10.1029/2011JA016479.

Koskinen, H. E. J., and E. I. Tanskanen (2002), Magnetospheric energy budget and the epsilon parameter, J. Geophys. Res., 107(A11), 1415, doi:10.1029/2002JA009283.

Lui, A. T. Y., M. W. Dunlop, H. Rème, L. M. Kistler, G. Gustafsson, and Q.-G. Zong (2007), Internal structure of a magnetic flux rope from Cluster observations, Geophys. Res. Lett., 34, L07102, doi:10.1029/ 2007GL029263.

Marghitu, O., et al. (2006), Experimental investigation of auroral generator regions with conjugate Cluster and FAST data, Ann. Geophys., 24(2), 619-635.

Marghitu, O., M. Hamrin, B. Klecker, K. Rönnmark, S. Buchert, L. M. Kistler, M. André, and H. Rème (2010), Cluster Observations of Energy Conversion Regions in the Plasma Sheet, pp. 453-459, Springer, Berlin, doi:10.1007/978-90-481-3499-1 32.

Miyashita, Y., et al. (2009), A state-of-the-art picture of substormassociated evolution of the near-Earth magnetotail obtained from superposed epoch analysis, J. Geophys. Res., 114, A01211, doi:10.1029/ 2008JA013225.

Nagai, T. (2006), Location of magnetic reconnection in the magnetotail, Space Sci. Rev., 122(1-4), 39-54, doi:10.1007/s11214-006-6216-4.

Nishida, A., H. Hayakawa, and E. Hones (1981), Observed signatures of reconnection in the magnetotail, J. Geophys. Res., 86(A3), 1422-1436.

Palmroth, M., H. E. J. Koskinen, T. I. Pulkkinen, P. K. Toivanen, P. Janhunen, S. E. Milan, and M. Lester (2010), Magnetospheric feedback in solar wind energy transfer, J. Geophys. Res., 115, A00110, doi:10.1029/2010JA015746. [Printed 116(A5), 2011.]

Paschmann, G., et al. (1979), Plasma acceleration at the earths magnetopause-Evidence for reconnection, Nature, 282(5736), 243-246.

Paschmann, G., S. Haaland, and R. Treumann (2002), Auroral Plasma Physics, Space Sci. Ser., vol. 15, Kluwer Acad., Dordrecht, Netherlands. Pulkkinen, T. I., M. Palmroth, and R. L. McPherron (2007), What drives magnetospheric activity under northward IMF conditions?, Geophys. Res. Lett., 34, L18104, doi:10.1029/2007GL030619.

Pulkkinen, T. I., M. Palmroth, and T. Laitinen (2008), Energy as a tracer of magnetospheric processes: GUMICS-4 global MHD results and observations compared, J. Atmos. Sol. Terr. Phys., 70(5), 687-707, doi:10.1016/ j.jastp.2007.10.011.

Reiff, P., and J. Burch (1985), IMF BY-dependent plasma-flow and Birkeland currents in the dayside magnetosphere: 2. A global model for northward and southward IMF, J. Geophys. Res., 90(A2), 1595-1609.

Rosenqvist, L., S. Buchert, H. Opgenoorth, A. Vaivads, and G. Lu (2006), Magnetospheric energy budget during huge geomagnetic activity using 
Cluster and ground-based data, J. Geophys. Res., 111, A10211, doi:10.1029/2006JA011608.

Rosenqvist, L., A. Vaivads, A. Retinò, T. Phan, H. J. Opgenoorth, I. Dandouras, and S. Buchert (2008), Modulated reconnection rate and energy conversion at the magnetopause under steady IMF conditions, Geophys. Res. Lett., 35, L08104, doi:10.1029/2007GL032868.

Scholer, M., B. Klecker, D. Hovestadt, G. Gloeckler, F. M. Ipavich, and E. J. Smith (1984), Fast moving plasma structures in the distant magnetotail, J. Geophys. Res., 89, 6717-6727, doi:10.1029/JA089iA08p06717.

Sergeev, V. A. (2005), Bursty bulk flows and their ionospheric footprints, in Multiscale Processes in the Earth's Magnetosphere: From Interball to Cluster, NATO Sci. Ser., vol. 178, edited by J.-A. Sauvaud and Z. Nemecek, pp. 289-306, Springer, Amsterdam, doi:10.1007/1-40202768-016.

Sharma, A. S., et al. (2008), Transient and localized processes in the magnetotail: A review, Ann. Geophys., 26(4), 955-1006.
Siscoe, G., and W. Cummings (1969), On cause of geomagnetic bays, Planet. Space Sci., 17(10), 1795-1802, doi:10.1016/0032-0633(69) 90055-5.

Slavin, J. A., M. F. Smith, E. L. Mazur, D. N. Baker, E. W. Hones Jr., T. Iyemori, and E. W. Greenstadt (1993), ISEE 3 observations of traveling compression regions in the Earth's magnetotail, J. Geophys. Res. 98(A9), 15,425-15,446, doi:10.1029/93JA01467.

Smith, C., J. L'Heureux, N. Ness, M. Acuña, L. Burlaga, and J. Scheifele (1998), The ACE magnetic fields experiment, Space Sci. Rev., 86, 613-632, doi:10.1023/A:1005092216668.

Tanaka, T., A. Nakamizo, A. Yoshikawa, S. Fujita, H. Shinagawa, H. Shimazu, T. Kikuchi, and K. K. Hashimoto (2010), Substorm convection and current system deduced from the global simulation, J. Geophys. Res., 115, A05220, doi:10.1029/2009JA014676.

Zong, Q.-G., et al. (2007), Earthward flowing plasmoid: Structure and its related ionospheric signature, J. Geophys. Res., 112, A07203, doi:10.1029/ 2006JA012112 